seroprevalence was higher, reaching $8.5 \%$ in Geneva University Hospital employees ${ }^{4}$ and $13.35 \%$ in a COVID-19-dedicated hospital in India. ${ }^{5}$ In these studies, the proportion of anti-SARSCoV-2 seroconversion was higher in employees working in COVID-19 areas. Risk factors included nosocomial outbreak and the use of public transportation. ${ }^{4}$ This last point was not evaluated in our study, but other nonprofessional risk factors, such as a contact with COVID-19 confirmed case, were not associated with a seroconversion.

In the urban area of the hospital around the same date, the raw SARS-CoV-2 seroprevalence was $2.1 \%$ among 2,006 individuals, ${ }^{6}$ suggesting the absence of high risk among laboratory staff, probably due to the strict application of the recommendations of the French Society of Microbiology (SFM) concerning sample handling. The benefit of correct use of personal protective equipment was also observed in HCWs, even in the most exposed groups. ${ }^{7}$

Despite the small size of the cohort and of self-reporting data collection, the work presented here originally targets SARSCoV-2-exposed laboratory staff. Today, most staff have been vaccinated, and studies evaluating the exposure of laboratory workers to a new airborne and/or hand-borne pathogen will no longer be possible. These data confirm the effectiveness of the good laboratory practices, which have to be quickly applied in future viral emergencies.

Acknowledgments. We thank all the laboratory staff who accepted to contribute to the study. We particularly thank the laboratory management staff, health executives, the staff of the Centre Regional de Traitement de l'Hemophilie and Dr Birgit Frotscher, and the young biologists that participated to sampling.
Financial support. No financial support was provided relevant to this article.

Conflicts of interest. All authors report no conflicts of interest relevant to this article.

\section{References}

1. Kea B, Johnson A, Lin A, et al. An international survey of healthcare workers use of personal protective equipment during the early stages of the COVID-19 pandemic. J Am Coll Emerg Physicians Open 2021;2:1-10.

2. Kasztelewicz B, Janiszewska K, Burzyńska J, Szydłowska E, Migdał M, Dzierżanowska-Fangrat $\mathrm{K}$. Prevalence of IgG antibodies against SARSCoV-2 among healthcare workers in a tertiary pediatric hospital in Poland. PLoS One. 2021;16:1-11.

3. Huang Q, Luo LS, Wang YY, Jin YH, Zeng XT. Gender differences in psychological and behavioral responses of infected and uninfected healthcare workers during the early COVID-19 outbreak. Front Public Heal 2021;9:1-8.

4. Martischang R, Iten A, Arm I, et al. SARS-CoV-2 seroconversion and occupational exposure of employees at a Swiss university hospital: a large longitudinal cohort study. Infect Control Hosp Epidemiol 2021;2:1-8.

5. Mahto M, Banerjee A, Biswas B, Kumar S, Agarwal N, Singh PK. Seroprevalence of IgG against SARS-CoV-2 and its determinants among healthcare workers of a COVID-19 dedicated hospital of India. Am J Blood Res 2021;11:44-52.

6. Gégout Petit A, Jeulin H, Legrand K, et al. Seroprevalence of SARS-CoV-2, symptom profiles and sero-neutralization in a suburban Area, France. Viruses. 2021;13:1076.

7. Caselli D, Loconsole D, Dario R, Chironna M, Aricò M. Effectiveness of preventive measures in keeping low prevalence of SARS-CoV-2 infection in healthcare workers in a referral children's hospital in southern Italy. Pediatr Rep 2021;13:118-124.

\title{
Healthcare-associated transmission of severe acute respiratory coronavirus virus 2 (SARS-CoV-2) among Thai healthcare personnel who receive 2 doses of a coronavirus disease 2019 (COVID-19) vaccine: A call for considering a booster dose
}

\author{
Anucha Apisarnthanarak MD , Sira Nantapisal MD, $\mathrm{PhD}^{2}$, Thanus Pienthong $\mathrm{MD}^{1}$, Piyaporn Apisarnthanarak $\mathrm{MD}^{3}$ and \\ David J. Weber MD, MPH \\ ${ }^{1}$ Division of Infectious Diseases, Department of Medicine, Thammasat University Hospital, Pathum Thani, Thailand, ${ }^{2}$ Division of Immunology, Department of \\ Pediatrics, Thammasat University Hospital, Pathum Thani, Thailand, ${ }^{3}$ Department of Radiology, Siriraj Hospital, Bangkok, Thailand and ${ }^{4}$ Gillings School of Global \\ Public Health, Chapel Hill, North Carolina, United States
}

To the Editor-There have been consistent reports of healthcare personnel (HCP) acquiring COVID-19 as a result of workplace exposure, either directly or indirectly. ${ }^{1,2}$ In Thailand, the emerging alpha variant of severe acute respiratory coronavirus virus 2 (SARS-CoV-2) replaced the original strain in February 2021, followed by the emergence of the delta variant of SARS-CoV-2 in

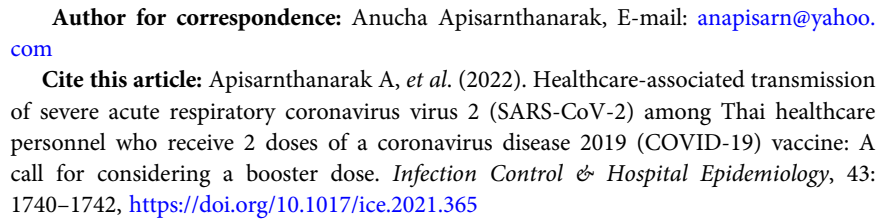

Cite this article: Apisarnthanarak A, et al. (2022). Healthcare-associated transmission of severe acute respiratory coronavirus virus 2 (SARS-CoV-2) among Thai healthcare personnel who receive 2 doses of a coronavirus disease 2019 (COVID-19) vaccine: A call for considering a booster dose. Infection Control \& Hospital Epidemiology, 43: 1740-1742, https://doi.org/10.1017/ice.2021.365

April 2021. ${ }^{3}$ Immunization of HCP was the first priority of the coronavirus disease 2019 (COVID-19) vaccination campaign, and most $\mathrm{HCP}$ received vaccine, based on the government vaccine allocation. As of July 19, 2021, CoronaVac (Sinovac-Biotech) and ChAdOx-1 (AstraZeneca) are the only 2 COVID-19 vaccines available in Thailand. Despite 2 doses of vaccine, the number of HCP who were infected with SARS-CoV-2 in Thailand is continuously increasing. To better understand the epidemiology of healthcare-associated SARS-CoV-2 transmission among HCP, we performed a retrospective review of HCP who received 2 doses of COVID-19 vaccine.

At Thammasat University Hospital a 650-bed, academic medical center in Pratum Thani, Thailand, a COVID-19 
vaccination campaign for HCP $(n=890)$ was initiated on February 1, 2021. Of 890 HCP, 860 (96.6\%) were vaccinated and were invited to participate in a vaccination serological study after vaccination. In total, $767 \mathrm{HCP}$ (89.2\%) received CoronaVac and 93 (10.8\%) received ChAdOx-1. Occupational health records from February 1, 2021, to July 16, 2021, were reviewed to evaluate the incidence of healthcare-associated SARS-CoV-2 transmission among HCP. Healthcare-associated SARS-CoV-2 transmission among HCP was defined as HCP who developed COVID-19 with a clear contact history to confirmed COVID-19 case(s) during patient care without using appropriate personal protective equipment (PPE) and without an epidemiology link to possible community or home transmission. Definitions of exposure risk and severity of symptoms are summarized in the Supplementary Material (online). ${ }^{4,5}$ Data collected included HCP demographics, underlying diseases, occupation, risk of exposures, severity of symptoms and serology after 2 doses of vaccinations (if available). Anti-spike receptor-binding domain antibody (anti-S-RBD-Ab) was measured using quantitative anti-SRBD IgG enzyme-linked immunosorbent assay (ELISA) in house to detect presumptive immunity to SARS-CoV-2 among vaccinated HCP who participated in the serology study, according to previously described techniques. ${ }^{6}$ The World Health Organization (WHO) standard for antiSARS-CoV-2 antibody (NIBSC code 20/136) was included in the assay as a reference standard curve. The optical density (OD) values from each serum sample were translated into anti-SRBD IgG levels (in BAU/mL) using the standard curve in the assay. Descriptive analysis was used to describe the characteristics of HCP who developed healthcare-associated COVID-19.

In total, 62 vaccinated HCP met the criteria for healthcare-associated COVID-19. Among 62 HCP 54 (87\%) were female; 38 (62\%) were nurses or nurse assistants; 16 (25.8\%) worked in the medicine department; and 42 (68\%) were categorized as having "high-risk" exposure (Table 1). Notably, 24 HCP (38.7\%) acquired COVID-19 via exposure to asymptomatic cases (Table 1). Furthermore, all infected HCP who had received 2 doses of COVID-19 vaccine developed mild COVID-19 symptoms that requiring admission by Thai law. Despite the vaccination campaign, a higher frequency of healthcare-associated SARS-CoV-2 transmission in our hospital was seen after the emergency of the alpha variants and was accelerated after the emergence of the delta variant of SARS-CoV-2 in Thailand (Supplement 1 online). Data showed that $8 \mathrm{HCP}$ had anti-S-RBD-Ab IgG prior to infection with COVID-19. The median anti-S-RBD-Ab IgG level for these $8 \mathrm{HCP}$ after vaccination was $112.5 \mathrm{BAU} / \mathrm{mL}$ (range, 25-355). The median anti-S-RBD-Ab IgG levels at day 60 after vaccination among HCP were $108.9 \mathrm{BAU} /$ $\mathrm{mL}$ for CoronaVac $(\mathrm{n}=7)$ versus $355 \mathrm{BAU} / \mathrm{mL}$ for ChAdOx-1 $(\mathrm{n}=1)$. The $7 \mathrm{HCP}$ who received CoronaVac were infected at a median of 67 days (range, 55-115) after the second vaccine dose, and 1 HCP who received 2 doses of ChAdOx-1 was infected 44 days after the second vaccine dose.

This study has some important implications. First, HCP were at extremely high risk for healthcare-associated SARS-CoV-2 transmission despite receiving 2 doses of COVID-19 vaccine. This risk may be explained by the suboptimal immune response following CoronaVac, particularly after day 60 . Based on our hospital serological study, HCP who received CoronaVac, tested after 60 days, had significantly lower antibody levels compared to those who were tested within 60 days of receiving CoronaVac $(111.1 \pm 62.63$ vs
Table 1. Healthcare Personnel Characteristics

\begin{tabular}{|c|c|}
\hline Variable & No. $(\%)(N=62)$ \\
\hline Age, median y, range & $34.4,21-67$ \\
\hline Sex, female vs male & $54(87.1)$ vs 8 (12.9) \\
\hline \multicolumn{2}{|l|}{ Underlying diseases } \\
\hline No & $51(82.3)$ \\
\hline Diabetes & $4(6.5)$ \\
\hline Hypertension & $3(4.8)$ \\
\hline Obesity & $2(3.2)$ \\
\hline Others $^{a}$ & $2(3.2)$ \\
\hline \multicolumn{2}{|l|}{ Department } \\
\hline Medicine & $16(25.8)$ \\
\hline Orthopedics & $11(17.7)$ \\
\hline Obstetrics and Gynecology & $5(8)$ \\
\hline Surgery & $4(6.5)$ \\
\hline Others $^{\mathrm{b}}$ & $26(41.9)$ \\
\hline \multicolumn{2}{|l|}{ Occupation } \\
\hline Nurses \& nurse assistants & $38(61.2)$ \\
\hline Doctors & $8(13)$ \\
\hline Others ${ }^{c}$ & $16(25.8)$ \\
\hline \multicolumn{2}{|l|}{ Exposure risk } \\
\hline High risk & $42(67.8)$ \\
\hline Intermediate risk & $20(32.2)$ \\
\hline Low risk & $0(0)$ \\
\hline $\begin{array}{l}\text { HCP exposure to index case wearing } \\
\text { inappropriate PPE }\end{array}$ & $42(67.8)$ \\
\hline HCP exposure to asymptomatic index case & $24(38.7)$ \\
\hline \multicolumn{2}{|l|}{ HCP symptoms } \\
\hline Asymptomatic & $0(0)$ \\
\hline Mild & $62(100)$ \\
\hline Moderate & $0(0)$ \\
\hline Severe & $0(0)$ \\
\hline
\end{tabular}

Note: No. (\%), unless indicated otherwise.

aUnderlying rheumatologic disease and immunocompromised state.

bepartment of Anesthesiology, Department of Ophthalmology, Department of Pediatrics, Department of Otolaryngology, Department of Radiology, Department of Dentistry,

Department of Pharmacology, Department of Nursing, Department of Physical Therapy, Supply Unit.

${ }^{c}$ Medical technologists, pharmacist assistants, physical therapists.

$237.4 \pm 160.4 \mathrm{BAU} / \mathrm{mL} ; P<.001$ ) (unpublished data). Antibody levels of HCP who completed 2 doses of ChAdOx-1 (12 weeks apart) at day 14 were measured at $401.8 \pm 289.1 \mathrm{BAU} / \mathrm{mL}$ (unpublished data). Together, these data suggest the need for a booster dose of COVID-19 vaccine among HCP, particularly those who received CoronaVac. Second, most HCP were categorized as having a high-risk exposure to the index case without using appropriate PPE. Third, we also noticed that a substantial proportion of HCP acquired SARS-CoV-2 from asymptomatic index cases. These data emphasize the need for continuous education to focus on the stringent use of PPE to enhance HCP safety, despite being fully vaccinated. Lastly, the fact that all HCP who were infected developed 
only mild COVID-19 symptoms confirmed the effectiveness of both vaccines to prevent severe disease and mortality. ${ }^{7,8}$

Our study had some limitations including the small sample size, the limited number of HCP who participated in a vaccination serological study after vaccination, and the possibility of misclassification bias from using epidemiology data to classified healthcare-associated SARS-CoV-2 transmission.

Additional studies to evaluate the viral transmission dynamic for the delta variant and its impact on healthcare-associated transmission among HCP who have completed different types of COVID-19 vaccine, as well as a longitudinal data regarding anti-S-RBD-Ab IgG among HCP, will provide insight into better protection of healthcare-associated transmission of COVID-19 among HCP.

\section{Acknowledgments.}

Financial support. No financial support was provided relevant to this article.

Conflicts of interest. All authors report no conflicts of interest relevant to this article.

Supplementary material. To view supplementary material for this article, please visit https://doi.org/10.1017/ice.2021.365

\section{References}

1. Nguyen LH, Drew DA, Graham MS, et al. Risk of COVID-19 among frontline healthcare workers and the general community: a prospective cohort study. Lancet Public Health 2020;5:475-483.

2. Chirico F, Nucera G, Magnavita N. COVID-19: protecting healthcare workers is a priority. Infect Control Hosp Epidemiol 2020;41:1117.

3. Corona virus diseases. Department of Disease Control website. https://ddc. moph.go.th/viralpneumonia/eng/index.php. Accessed July 4, 2021.

4. Corona virus information. McGill website. https://www.mcgill.ca/coronavirus/ health-guidelines/potential-covid-19-exposure-what-do. Accessed July 4, 2021.

5. Coronavirus diseases 2019 (COVID-19) treatment guidelines. National Institutes of Health website. https://www.covid19treatmentguidelines.nih. gov/. Accessed July 4, 2021.

6. Amanant E, Stadlbauer D, Strohmeier S, et al. A serological assay to detect SARS-CoV-2 seroconversion in humans. Nat Med 2020;26: 1033-1036.

7. Palacios R, Patiño EG, de Oliveira Piorelli R, et al. Double-blind, randomized, placebo-controlled phase III clinical trial to evaluate the efficacy and safety of treating healthcare professionals with the adsorbed COVID-19 (inactivated) vaccine manufactured by Sinovac_-PROFISCOV: a structured summary of a study protocol for a randomised controlled trial. Trials 2020;21:853.

8. Knoll MD, Wonodi C. Oxford-AstraZeneca COVID-19 vaccine efficacy. Lancet 2021;397:72-74.

\title{
Transmission of severe acute respiratory coronavirus virus 2 (SARS-CoV-2) among health careworkers (HCWs) during three waves of the coronavirus disease 2019 (COVID-19) pandemic in Germany: Results of an anonymous survey
}

\author{
Prof. Dr. med. Sebastian Schulz-Stübner ${ }^{1}$ and Elke Pielert ${ }^{2}$ \\ ${ }^{1}$ German Consulting Center for Infection Prevention and Control [Deutsches Beratungszentrum für Hygiene (BZH GmbH)], Freiburg, Germany and ${ }^{2} \mathrm{Krankenhaus}$ \\ St. Elisabeth und St. Barbara Halle (Saale) GmbH, Halle (Saale), Germany
}

To the Editor-In an editorial in the Journal of the American Medical Association (JAMA), Gohil and Huang postulated the following: "Healthcare personnel (HCP) have absorbed substantial risks of acquiring coronavirus disease 2019 (COVID-19) due to their care of patients with COVID-19 infection throughout the pandemic. Nevertheless, because of robust health care infection prevention and control practices and policies that prevent patient-to-HCP transmission, it is possible that the greatest risk of COVID-19 transmission to HCP comes from exposure in their communities and, secondarily, between essential workers."

To learn more about the transmission of severe acute respiratory coronavirus virus 2 (SARS-CoV-2) among healthcare workers (HCWs), we conducted an anonymous survey that was distributed by mass e-mail through interested infection control practitioners to all employees in their respective institution. The survey sheets

Author for correspondence: Prof. Dr. Sebastian Schulz-Stübner, E-mail: Schulzstuebner@bzh-freiburg.de

Cite this article: Schulz-Stübner PDS and Pielert E. (2022). Transmission of severe acute respiratory coronavirus virus 2 (SARS-CoV-2) among health careworkers (HCWs) during three waves of the coronavirus disease 2019 (COVID-19) pandemic in Germany: Results of an anonymous survey. Infection Control \& Hospital Epidemiology, 43: 1742-1744, https://doi.org/10.1017/ice.2021.359 were printed out and sent without sender information by mail to a central collecting address to guarantee that no backward data tracking was possible. No personal identifying data were collected, in accordance with German General Data Protection Regulation (GDPR). Because this was not a human-subject study, no ethics committee review was needed.

Because of the completely anonymous data collection, we do not know how many surveys were distributed. In total, 116 survey sheets were returned, and 1 was excluded because of implausible data. Table 1 shows the detailed results which clearly identify the 3 COVID-19 waves in Germany during the periods evaluated in the study. In total, 17 cases with mild or no symptoms and short duration were breakthrough infections during the third wave; however, only 2 were fully vaccinated according to the current definition ( $>14$ days after the second shot of the respective vaccine).

Overall, $14 \%$ of the 115 cases were attributed to private contacts, $8 \%$ were undetermined and $78 \%$ were attributed to professional contacts. In addition, 54 (60\%) of those 90 cases were related to unprotected contact of $>15$ minutes to undiagnosed patients or colleagues and $18(20 \%)$ to aerosol-generating procedures (AGPs). Among those 18 AGP cases, free-text comments indicated 3 cases involving endotracheal intubation and 1 case 